\title{
R726L Androgen Receptor Mutation Is Uncommon in Prostate Cancer Families in the United States
}

\author{
Stephen B. Gruber, ${ }^{1,3}$ Hong Chen, ${ }^{1}$ Lynn P. Tomsho, ${ }^{1}$ Nana Lee, ${ }^{1}$ \\ Erin E. Perrone, ${ }^{2}$ and Kathleen A. Cooney ${ }^{1,2,4 *}$ \\ 'Department of Internal Medicine, University of Michigan Medical School, Ann Arbor, Michigan \\ ${ }^{2}$ Department of Urology, University of Michigan Medical School, Ann Arbor, Michigan \\ ${ }^{3}$ Department of Epidemiology, University of Michigan School of Public Health, Ann Arbor, Michigan \\ ${ }^{4}$ Department of Veterans Affairs Medical Center, Ann Arbor, Michigan
}

\begin{abstract}
BACKGROUND. A mutation in the androgen receptor $(A R)$ gene, namely $A R$ R726L, was described in $2 \%$ of Finnish men with sporadic or familial prostate cancer and was associated with an approximately sixfold increased risk of prostate cancer. We set out to determine the incidence of this mutation in a sample of men with either early-onset and/or familial prostate cancer in the United States.

METHODS. Five hundred forty-eight men with prostate cancer from 411 unrelated families participating in the University of Michigan Prostate Cancer Genetics Project (PCGP) were studied. Allele-specific oligonucleotide hybridization was used to detect the presence of the $A R$ R726L mutation in germline DNA.

RESULTS. None of the 548 prostate cancer patients studied, including 513 White, 29 African American, 3 Asian, and 3 Hispanic men, were found to carry the AR R726L allele. Therefore, the prevalence of this allele is significantly less than that observed among Finnish men with prostate cancer (Fisher's exact test, $P=0.002$ ).

CONCLUSIONS. The AR R726L allele does not account for a significant proportion of earlyonset and/or familial prostate cancer in the United States. Prostate 54: 306-309, 2003.
\end{abstract}

(C) 2003 Wiley-Liss, Inc.

KEY WORDS: prostate carcinoma; genetic susceptibility; founder mutation

\section{INTRODUCTION}

The observation that prostate cancer aggregates in families has led investigators to search for germline mutations that may increase the susceptibility to prostate cancer (see review [1]). Since the relative risk attributed to having a brother with prostate cancer is twice as high as that conferred by an affected father, some investigators have suggested that there may be $\mathrm{X}$-linked and/or recessive prostate cancer loci [2]. The androgen receptor $(A R)$ gene (OMIM *313700), located at $\mathrm{Xq} 11-12$, has been proposed as a candidate prostate cancer susceptibility gene. However, the $A R$ gene is approximately $50 \mathrm{cM}$ from the major HPCX candidate region, and family based linkage studies have not demonstrated linkage to the $A R$ gene [3].
Recently, Mononen et al. [4] reported that a germline mutation in the $A R$ gene, specifically R726L in exon E, occurs in approximately $2 \%$ of Finnish men with prostate cancer. This mutation was observed in a similar

\footnotetext{
Abbreviations: AR, androgen receptor; hAR, human androgen receptor protein; OMIM, online Mendelian inheritance in man; ASO, allele specific oligonucleotide hybridization; $\mathrm{dx}$, diagnosed.

Grant sponsor: USPHS; Grant numbers: P50-CA69568, R01CA79596, R01-CA81488.

*Correspondence to: Kathleen A. Cooney, MD, 7310 CCGC, 1500 East Medical Center Drive, Ann Arbor, MI 48109-0946.

E-mail: kcooney@umich.edu

Received 26 April 2002; Accepted 12 September 2002

DOI $10.1002 /$ pros.10195
} 
frequency in men with both familial and sporadic prostate cancer, but it was detected in only $0.33 \%$ of $A R$ alleles among healthy blood donors. Overall, an approximately sixfold increased risk of prostate cancer was attributed to the R726L substitution $(\mathrm{OR}=5.8,95 \%$ $\mathrm{CI}=1.6-21.2)$. In 11 out of 13 men who carried the mutation, the R726L substitution occurred in an $A R$ allele containing $26 \mathrm{CAG}$ repeats suggesting the presence of a founder allele in the Finnish population.

The University of Michigan Prostate Cancer Genetics Project (PCGP) is a family-based study that was established to determine the molecular basis for inherited prostate cancer susceptibility. We set out to determine the potential contribution of the $A R$ R726L mutation to familial prostate cancer in an ethnically diverse population from the US.

\section{METHODS}

\section{Study Population}

Five hundred forty-eight men with histologically confirmed prostate cancer from 411 unrelated families who are participants in the University of Michigan PCGP were included in the study. Men with prostate cancer are eligible to enroll in the PCGP if they were diagnosed $\leq$ age 55 and/or if they have $\geq 1$ living firstor second-degree relative with prostate cancer. Written, informed consent was obtained from each patient, and the study protocol was approved by the Institutional Review Board of the University of Michigan. Two hundred seventy of the men were from families with two or more affected men with prostate cancer; these men were described previously in an analysis of $A R$ CAG length [5]. All diagnoses of prostate cancer were confirmed by pathology reports or review of medical records.

\section{Laboratory Methods}

Genomic DNA was isolated from whole blood using a commercially available kit (Puregene DNA extraction kit; Gentra Systems, Inc., Research Triangle, NC). Allele-specific oligonucleotide (ASO) hybridization was performed as previously described [4]. Male genomic DNA containing the AR R726L mutation was used as a hybridization control (kindly provided by N. Mononen). AR CAG repeat length was measured as previously reported.

\section{Statistical Methods}

Contingency tables were analyzed using Fisher's exact test, and all $P$-values are reported as two-tailed tests. All analyses were conducted using SAS version 8.00 (SAS Institute, Inc., Cary, NC).

\section{Protein Modeling}

Diagrams of the three-dimensional structure of the ligand-binding domain (LBD) of the human androgen receptor protein (hAR) were built using RIBBONS 2.0 [6] based on the coordinates of the hAR LBD-R1881 complex [7] available through the Protein Data Bank (PDB ID 1e3g, http:/ / www.rcsb.org) [8]. The hAR LBD consists of 262 amino acids encompassing amino acids 657 through 919 of the full-length AR.

\section{RESULTS}

In this study, germline DNA was analyzed from a total of 548 men with prostate cancer, including 411 unrelated probands with prostate cancer. The average age of prostate cancer diagnosis in the men reported here was 61.0 years $(\mathrm{SD}=9.6$, range $36-93$ ). Forty percent of probands were diagnosed $\leq$ age 55 . Although the majority of participants described themselves as white and not of Hispanic origin, our sample included 29 African American men, 3 Asian men, and 3 Hispanic men.

Using ASO hybridization, none of the 548 alleles analyzed were shown to contain the R726L mutation (Fig. 1). Therefore, the prevalence of the AR R726L mutation among either the entire set of 548 US men with prostate cancer or the 411 probands was significantly less than that reported in Finnish men with prostate cancer (Table I, Fisher's exact test, $P<0.0001$ and $P=0.003$, respectively). When the PCGP probands were restricted to those diagnosed at or before age 55 , the prevalence of the $A R$ R726L substitution in men with prostate cancer was also significantly less than that detected in the Finnish population (Table I, Fisher's exact test, $P=0.035$ ). Furthermore, there was a significant difference in the incidence of the R726L mutation between white men with prostate cancer from the PCGP and Finnish prostate cancer cases (Fisher's exact test, $P=0.002$ ).

\section{DISCUSSION}

The AR R726L mutation is an interesting candidate prostate cancer susceptibility allele, and previous functional studies have demonstrated that this substitution results in an $A R$ that is activated by both testosterone and estradiol [9]. Three-dimensional modeling of hAR LBD in complex with the ligand metribolone (R1881) [7] shows the side chain of R726 protruding out into the surrounding milieu (Fig. 2). Since the location of this amino acid is at a distance from the ligand-binding site, the R726L substitution is unlikely to directly alter binding affinity. The R726L polymorphism is also not predicted to have a significant impact on overall structure. However, substitution of a nonpolar amino 

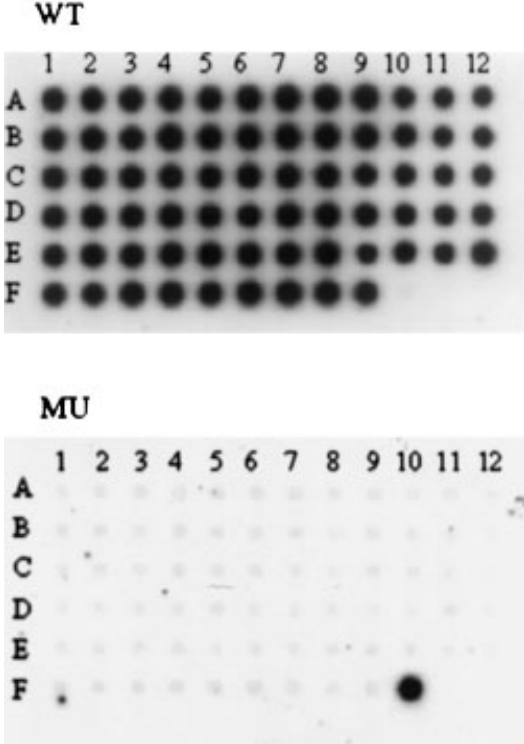

Fig. I. Allele-specific oligonucleotide (ASO) hybridization for detecting the AR R726L substitution. All wells contained DNA samples from men with prostate cancer participating in the Prostate Cancer Genetics Project except well FIO which contained control DNA from a male carrier of the AR R726L allele and wells FII and FI2, which were water blanks. Duplicate blots were hybridized with the wild-type oligonucleotide in the panel labeled wild type or with the R726L oligonucleotide in the panel labeled mutant.

acid (leucine) for a basic amino acid (arginine) may alter AR surface charge and consequently influence other molecular interactions, such as with coactivators of receptor action.
Exon 1 of the $A R$ gene contains a highly polymorphic trinucleotide (CAG) repeat sequence, and shorter AR CAG repeats have been suggested to increase the risk of prostate cancer in some studies [10-12]. This relationship is biologically plausible since in vitro experiments reveal that $A R$ alleles with fewer CAG repeats result in an $A R$ with increased transactivation function [13]. In the Mononen report, however, $11 / 13$ of the Finnish prostate cancer patients with the $A R$ R726L mutation also had an AR CAG repeat length of 26 consistent with linkage disequilibrium between the AR CAG repeat and the R726L mutation [4]. Since short rather than long CAG repeats have been associated with prostate cancer incidence, the CAG repeat of 26 is unlikely to be contributing to the risk of prostate cancer associated with this Finnish AR allele.

In our study, we considered two possible hypotheses. First, the AR R726L allele could be a founder allele in the US in which case the mutation should be detected on an $A R$ allele containing a CAG repeat length of 26. Alternatively, the AR R726L mutation could be occurring de novo in the population, and thus the R726L mutation should be detected on an AR allele with any number of CAG repeats. The most common number of CAG repeats in Caucasian men with prostate cancer in the US is 21 , and alleles with more than 25 CAG repeats or fewer than 19 repeats are relatively uncommon [11]. Since we failed to detect the R726L mutation in our study population, we reviewed our data with regard to $A R$ CAG length that was available from a subset of the men in our report [5]. Although AR alleles with 26 CAG repeats are relatively

TABLE I. Prevalence of the R726L Androgen Receptor Mutation in US Prostate Cancer Patients

\begin{tabular}{lrccc}
\hline & \multicolumn{4}{c}{ Genotype } \\
\cline { 2 - 5 } & $\mathrm{N}$ & R726L & Wild Type & $\%$ \\
\hline University of Michigan PCGP & 548 & 0 & 548 & 0 \\
White (not Hispanic origin) & 513 & 0 & 513 & 0 \\
African American & 29 & 0 & 29 & 0 \\
Asian or Pacific Islander & 3 & 0 & 3 & 0 \\
Hispanic & 3 & 0 & 3 & 0 \\
University of Michigan PCGP & 411 & 0 & 411 & 0 \\
probands & & & & \\
Probands dx $\leq 55$ & 163 & 0 & 248 & 0 \\
Probands dx $>55$ & 248 & 0 & & \\
Finland & 1,302 & & 410 & 1.91 \\
Sporadic prostate cancer & 418 & 8 & 104 & 1.92 \\
Familial prostate cancer & 106 & 2 & 897 & 0.33 \\
Healthy blood donors & $900^{\mathrm{b}}$ & 3 & & \\
\hline
\end{tabular}

${ }^{\mathrm{a}}$ Data from Mononen et al. [4].

${ }^{\mathrm{b}}$ Results from 656 male and 122 female, healthy, anonymous blood donors for a total of $900 \mathrm{X}$ linked $A R$ alleles examined. 


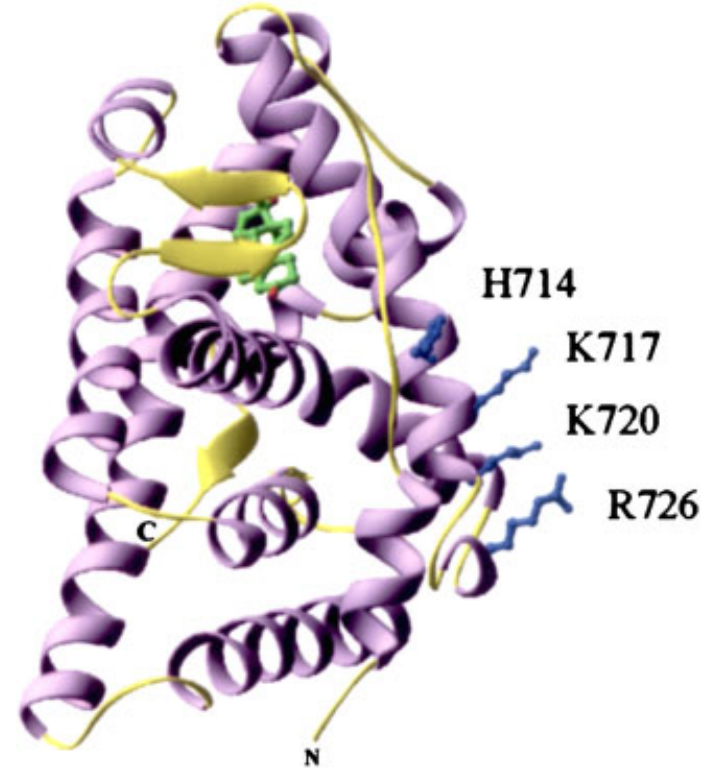

Fig. 2. RIBBONS 2.0 diagrams of the three-dimensional structure of hAR LBD-RI88I. The basic residues H7I4, K7I7, R720, R726 are indicated in blue. The ligand metribolone (RI88I) is indicated in green.

rare, at least 11 men in this study (from 11 unrelated families) were shown to carry an $A R$ allele containing a CAG repeat length of 26 .

The minimum number of independent $A R$ alleles in this data set is 411 taking into account familial relationships. We found no evidence of the R726L mutation in white men with prostate cancer from our study of inherited prostate cancer susceptibility in the US compared to Finnish cases. In addition, we did not find the mutation in any of the African American, Asian/Pacific Islander, or Hispanic men with prostate cancer in our study (Table I). However due to small sample sizes, our power was too low to detect this mutation in any racial subgroup other than white men.

\section{CONCLUSIONS}

Our data are consistent with the hypothesis that the $A R$ R726L allele is a founder prostate cancer allele in Finland, and that this mutation does not account for a significant proportion of early-onset and/or familial prostate cancer in the United States. These data also suggest that population stratification remains an important methodological issue in studies of the genetic epidemiology of prostate cancer.

\section{ACKNOWLEDGMENTS}

We thank D. Robins for helpful discussions of androgen receptor function and J. Stuckey, Laboratory for the Analysis of Macromolecular Structure, for assistance with the protein modeling.

\section{REFERENCES}

1. Ostrander EA, Stanford JL. Genetics of prostate cancer: Too many loci, too few genes. Am J Hum Genet 2000;67:1367-1375.

2. Monroe KR, Yu MC, Kolonel LN, Coetzee GA, Wilkens LR, Ross RK, Henderson BE. Evidence of an X-linked or recessive genetic component to prostate cancer risk. Nat Med 1995;1:827829.

3. Lange EM, Chen $\mathrm{H}$, Brierley $\mathrm{K}$, Perrone EE, Bock $\mathrm{CH}$, Gillanders E, Ray ME, Cooney KA. Linkage analysis of 153 prostate cancer families over a $30-\mathrm{cM}$ region containing the putative susceptibility locus HPCX. Clin Cancer Res 1999;5: 4013-4020.

4. Mononen N, Syrjakoski K, Matikainen M, Tammela TL, Schleutker J, Kallioniemi OP, Trapman J, Koivisto PA. Two percent of Finnish prostate cancer patients have a germ-line mutation in the hormone-binding domain of the androgen receptor gene. Cancer Res 2000;60:6479-6481.

5. Lange EM, Chen $\mathrm{H}$, Brierley $\mathrm{K}$, Livermore $\mathrm{H}$, Wojno $\mathrm{KJ}$, Langefeld CD, Lange K, Cooney KA. The polymorphic exon 1 androgen receptor $C A G$ repeat in men with a potential inherited predisposition to prostate cancer. Cancer Epidemiol Biomarkers Prev 2000;9:439-442.

6. Carter M. Ribbons 2.0. J Appl Crystallogr 1991;24:958-961.

7. Matias PM, Donner P, Coelho R, Thomaz M, Peixoto C, Macedo S, Otto N, Joschko S, Scholz P, Wegg A, Basler S, Schafer M, Egner U, Carrondo MA. Structural evidence for ligand specificity in the binding domain of the human androgen receptor. Implications for pathogenic gene mutations. J Biol Chem 2000;275:26164-26171.

8. Berman HM, Westbrook J, Feng Z, Gilliland G, Bhat TN, Weissig H, Shindyalov IN, Bourne PE. The protein data bank. Nucleic Acids Res 2000;28:235-242.

9. Elo JP, Kvist L, Leinonen $\mathrm{K}$, Isomaa $\mathrm{V}$, Henttu $\mathrm{P}$, Lukkarinen $\mathrm{O}$, Vihko P. Mutated human androgen receptor gene detected in a prostatic cancer patient is also activated by estradiol. J Clin Endocrinol Metab 1995;80:3494-3500.

10. Irvine RA, Yu MC, Ross RK, Coetzee GA. The CAG and GGC microsatellites of the androgen receptor gene are in linkage disequilibrium in men with prostate cancer. Cancer Res 1995;55: 1937-1940.

11. Giovannucci E, Stampfer MJ, Krithivas K, Brown M, Brufsky A, Talcott J, Hennekens CH, Kantoll PW. The CAG repeat within the androgen receptor gene and its relationship to prostate cancer. Proc Natl Acad Sci USA 1997;94:3320-3323.

12. Stanford JL, Just JJ, Neal CL, Blumenstein BA, Ostrander EA. Polymorphic repeats in the androgen receptor gene: Molecular markers of prostate cancer risk. Cancer Res 1997;57:1194-1198.

13. Chamberlain NL, Driver ED, Miesfeld RL. The length and location of CAG trinucleotide repeats in the androgen receptor N-terminal domain affect transactivation function. Nucleic Acids Res 1994;22:3181-3186. 